\title{
Development of a Self-Healing Strategy With Multiagent Systems for Distribution Networks
}

\author{
Jônatas Boás Leite, Member, IEEE, and José Roberto Sanches Mantovani, Member, IEEE
}

\begin{abstract}
The increase in information and communication technologies in the electric power system has led the distribution network to a new evolutionary stage known as a smart grid. In this scenario, automatic local switching plans based on an understanding of the network topology and load behavior allow the implementation of self-healing strategies. Self-healing aims at the minimization of the number of switching operations within the shortest time interval to restore the maximum load affected by a fault and to preserve distribution network operation limits. In this paper, multiagent system concepts are employed to develop a self-healing strategy, where all switching local agents have some awareness regarding the current network state and work cooperatively to reach the global purpose of distribution network automatic restoration.
\end{abstract}

Index Terms-Distribution network, smart grid, self-healing, multiagent systems.

\section{INTRODUCTION}

$\mathbf{E}$ VEN AFTER the evolutionary stage of the smart grid, transmission lines, distribution feeders, switches, circuit breakers and transformers still remain as the core equipment of the transmission and distribution (T\&D) system. Intelligence in the T\&D system has been incorporated by advances in the monitoring, control and protection of the core equipment. The basic protection and automation schemes have changed to distributed and communicative architectures where system operation applications are more advanced in their ability to coordinate distributed intelligence on substations and feeders by ensuring high indices of reliability, efficiency and security. Those operational advances originate in the selfhealing applications that minimize service disruption through the employment of modern technologies that can acquire data, execute decision-support algorithms, avert or limit interruptions, control the power flow dynamically and restore service quickly [1].

Manuscript received July 13, 2015; revised December 4, 2015; accepted January 11, 2016. Date of publication January 28, 2016; date of current version August 21, 2017. This work was supported in part by the São Paulo Research Foundation under Grant 2014/22377-1, Grant 2013/23590-8, and Grant 2015/17757-2; in part by the Coordenação de Aperfeiçoamento de Pessoal de Nível Superior; and in part by the Conselho Nacional de Desenvolvimento Científico e Tecnológico under Grant 305371/2012-6. Paper no. TSG-00814-2015.

The authors are with the Electrical Engineering Department, São Paulo State University, Ilha Solteira 15385-000, Brazil (e-mail: jonatasboasleite@gmail.com; mant@dee.feis.unesp.br).

Color versions of one or more of the figures in this paper are available online at http://ieeexplore.ieee.org.

Digital Object Identifier 10.1109/TSG.2016.2518128
In the literature, different ways of implementing self-healing networks are described in [2] and [3], which also propose a hybrid architecture by combining centralized and distributed intelligence. Smart switching devices, communication systems and automatic restoration logic synthesize the hybrid nature of self-healing, and they are the successful keys of many distribution network projects, as assessed by [4]. In this paper, the keys to successful self-healing are followed using the technique of distributed artificial intelligence (DAI) to combine adaptive protection schemes with automatic restoration algorithms.

DAI is defined as the study, construction and application of multiagent systems (MAS) where several intelligent agents interact with each other to reach a set of goals or to perform a set of tasks [5]. Intelligent agents should take flexible and autonomous actions to meet their design objectives. The agent flexibility is composed of reactivity, pro-activeness and social ability [6]. Therefore, MAS represents the best way to characterize and design distributed computational systems [7] that are required by self-healing networks.

In [8], a control structure with cooperative MAS for selfhealing operation is proposed. This structure has two layers: zone and feeder. The zone agents monitor the distribution network sections that are limited by protective devices, make simple calculations and take control actions. The feeder agents are engaged in the negotiations through two-way communication. Thus, after the circuit breaker tripping, the feeder agent closest to the fault must localize and isolate the affected section while other agents aid in the restoration procedure. A resembling structure is proposed by [9], where the MAS is divided into three layers: response, coordination and organization. The response layer is composed of some modules of the agents with essential functions such as data pre-processing, fault location, topology analysis, reconfiguration and restoration. The coordination layer has agents of emergency, restorative, corrective and preventive control that coordinate the related agents to enable their control actions. The organization layer has a self-healing control agent that obtains practical data to initiate the coordination agent correlated with required control action. Ding and Loparo [10] assume that quick and efficient reconfiguration is indispensable for implementing an autonomous electrical network with self-healing features. Thus, they propose a hierarchical and decentralized MAS architecture where agents are responsible for the reconfiguration of subnets that are sections of the 
decomposed electrical network. Coordination among agents is crucial in this architecture because each agent should collect the local information, solve the local reconfiguration and decide the control action.

Quoted MAS were proposed as a computational tool of the operation center where the employment of MAS aims to reduce the processing time for automatic restoration algorithms. Furthermore, agents only perform their internal functions after the circuit breaker tripping; consequently, there is a significant time interval between circuit breaker disarming and the restoration of the loads affected by the fault due to the processing time of the centralized MAS.

The European technological platform for smart grids defines the self-healing concept as the automatic restoration strategies of electrical networks with a high level of decentralized preventive control methodologies. In this way, the development of a decentralized MAS is proposed where the protection devices are addressed as distributed intelligent agents that adapt to the electrical network changes for a certain time. Intelligent agents in the distribution network can communicate via the IEC 61850 protocol, which has a structure subscriber/publisher suitable for MAS. Through the communication mean, distributed agents become aware of the electrical network's state and actuate locally, aiming at a global action in a cooperative way that requires the employment of coordinator agents. Therefore, the proposed MAS control structure and the embedded algorithms of the distributed and coordinator agents are valuable for the solution to the self-healing problem. Regarding this paper's structure, Section II presents the proposed MAS architecture for the self-healing strategy using a smart grid reference model that permits the description of the communication protocol among agents and the formulations of the switching local agent and of the self-healing coordinator agent. Section III shows a case study with responses produced by the agents. Section IV contains the conclusions together with the main advances of the proposed method.

\section{MAS Architecture of Self-Healing Strategy}

The modeling methods of interactions among agents, which guarantee an intelligent global response to some system tasks, continue to mature once there is not a consensus regarding the suitable coordination level among agents. On the one hand, a special coordinator agent can be designed to establish interdependencies among activities of local agents using successive abstraction levels. On the other hand, agents can be designed to interact laterally without the requirement of a special agent. The first coordination model produces a hierarchical integration of control planes determined by high-level functions, whereas the second model favors the integration according to the social knowledge of agents. Although both approaches are feasible, the first approach is more reliable for system operation [11].

Self-healing applications should anticipate and respond to system disturbances through the performance of continuous self-assessment to detect, analyze and take corrective actions against dangerous events. Thus, the hierarchical coordination model fits perfectly with the requirements of self-healing

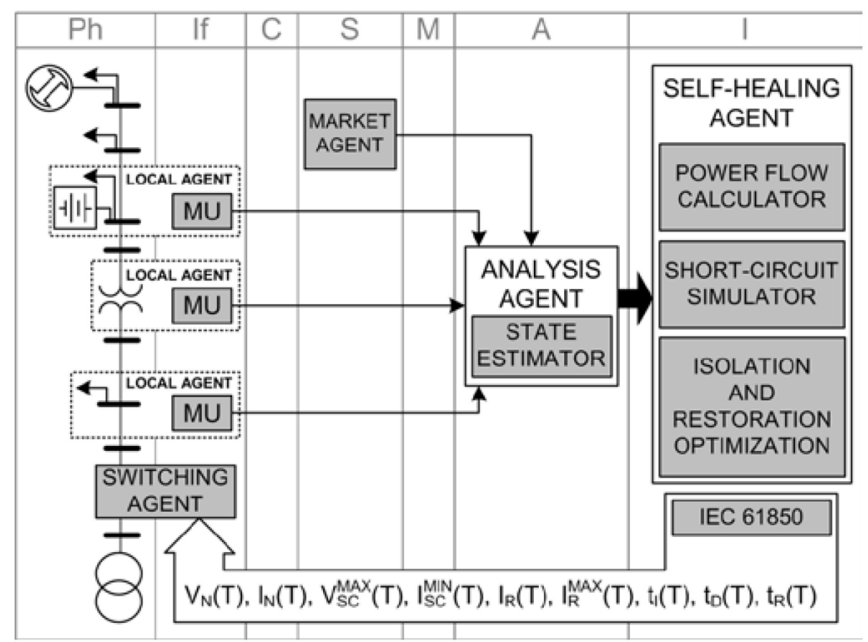

Fig. 1. Self-healing information flow in the layered architecture of smart grid.

functionalities. Furthermore, the layered architecture of the smart grid, which is shown in Fig. 1 and employed to integrate the electrical power grid with communication and information technologies [12], allows for expected continuous self-assessment through different control planes. The information flow is two-way and can be represented by a cycle, where the metering data are sent to the state estimator and, afterwards, they return as control parameters of the switching agents, which isolates and restores the distribution network within a fraction of a second.

The monitoring and controlling processes constitute the cycle that repeats in every time interval $T$ and begins with the measurement of the electrical magnitudes, such as current flow and the voltage profile, from the physical layer $(\mathrm{Ph})$ viewed as the MAS environment. Measurements are taken using merging units (MU) of local agents that are distributed along an electrical network and placed at the interface layer (If). In the upper layers, there are more sophisticated agents with specific functions to aggregate and process data from distributed agents in the lower layers. For example, the analysis agent receives from the local and market agents, respectively, the metering data and the energy market data that are used in the realtime state estimation of the distribution network. The values of network loading are transmitted to the intelligence layer (I), where specialized algorithms such as power flow, short-circuit and restoration optimization are employed by the self-healing agent to coordinate the local agents, especially the switching agents.

The power flow algorithm obtains the normal condition of operation for the time period $T$ through the computation of the current flow $I_{N}(T)$ and the voltage profile $V_{N}(T)$, whereas the short-circuit algorithm simulates the minimum current, $I_{S C}^{M I N}(T)$ and the maximum voltage $V_{S C}^{M A X}(T)$ for the abnormal condition of operation. The coordination algorithm uses all computed and simulated values to coordinate the smart switching agents through the calculation of the isolation time $t_{I}(T)$, delayed isolation time $t_{D}(T)$, and restoration time $t_{R}(T)$; moreover, restoration optimization also specifies the maximum restored load through the determination of the restored 
current $I_{R}(T)$. Finally, the cycle completes when switching agents receive all updated control parameters.

The core of the proposed self-healing strategy is composed of a MAS architecture where the self-healing coordinator agent works as the consciousness of the switching local agents, modifying their perception according to their beliefs and design objectives. Thus, the success of this architecture depends on the communication protocol among agents.

\section{A. IEC 61850 Standard as Agent Communication Protocol}

The elaboration of the IEC 61850 protocol began in 1990 with the purpose of introducing a communication standard that could solve the problem of different protocols used in the power system. Its development was based on the interoperability of intelligent electronic devices (IED) from different manufacturers. Indeed, the standard does not determinate the allocation of device functions; it only specifies the structure and communication interface of the functions through the definition of important details for interoperability. Thus, different vendors can continue developing new functionalities because the IEC 61850 standard does not confine the development of IEDs or substation automation [13].

Fig. 2(a) illustrates the object of the IEC 61850 standard that is the logical representation of a physical device, where the memory positions and device functions are decomposed in small logical entities. Fortunately, this standard is not rigid and permits the developers to insert automation codes into the IEDs. If the added automation has autonomy algorithms, then the IEDs can be addressed as agents, as shown in Fig. 2(b). In accordance with this viewpoint, the IEC 61850 standard has the needed ontology for communication among agents. A careful investigation [14] also reveals the satisfaction of other requirements, such as:

1) Communication Network Scheme: the concept of the Abstract Communication Service Interface (ACSI) provides two communication services: a client/server model to obtain data from IEDs, and a peer-to-peer model for fast communication and periodic transmission.

2) Performative Language: the performative characteristic is obtained by the utilization of declarative GOOSE messages and directive ACSI services such as the control model.

3) Agency Coordination Architecture: the three different levels (station, bay/unit and process) permit the development of hierarchical MAS, where the coordinator agent can be placed at station level or above. Furthermore, probable agents in the bay level can interact laterally with decentralized coordination.

The IEC 61850 standard provides resources that guarantee information exchange among switching local agents and the self-healing coordinator agent.

\section{B. Switching Local Agent}

Local agents placed at the interface layer actuate in the physical layer, e.g., opening/closing of a circuit breaker to control the energy flow and exchanging information with agents from upper layers. Their autonomy comprises the

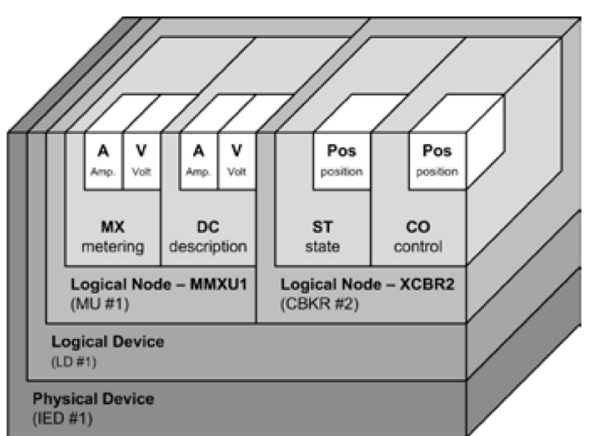

(a)

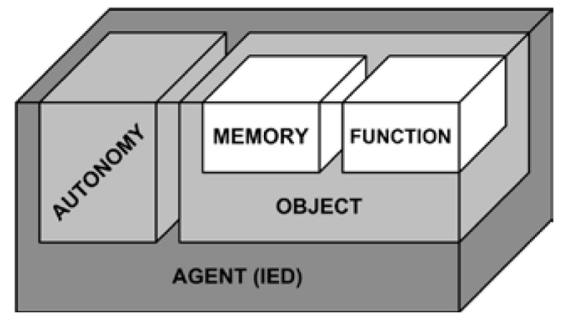

(b)

Fig. 2. Logical representation of an IED: (a) IEC 61850 object; and (b) expansion to agent.

knowledge base and the inference mechanism. The knowledge base is achieved by a prior knowledge added to past experience/data that makes more efficient inference mechanism calculations. The inference mechanism, in turn, uses the knowledge base information for its observations, abilities, beliefs and goals to produce an action or decision making [15].

The addition of the fuzzy controller into the switching local agent supplies the requirements of artificial intelligence because the system of fuzzy rules also comprises a knowledge base and an inference mechanism. Moreover, the fuzzy logic is linguistic, variables are used seemly for human thinking, and it has robustness and simplifies knowledge acquisition and representation [16]. Fuzzy controller responses are processed using observations collected from the environment within a fraction of a second for allowing real-time operation, whereas the prior knowledge base can be adapted to new environment conditions according to information from the coordinator agent.

The switching local agent communicates with other agents and monitors instantaneous values such as current and voltage magnitudes that are employed by the fuzzy controller in the inference procedure. Fig. 3 illustrates four charts of membership functions resulting from the fuzzification of current magnitude, in (a), voltage magnitude, in (b), time signal, in (c), and command signal, in (d). Current membership functions comprise the two-way power flow, i.e., the direct power flow $(i>0)$ and the reverse power flow $(i<0)$, which occur in the restorative condition of the distribution network. In the second chart, the voltage membership function of poor magnitude is between voltage membership functions of short circuits and normal operation. In the third chart, time membership functions of isolation and restoration cover the same time interval that is limited by the time period of transitory events and by 


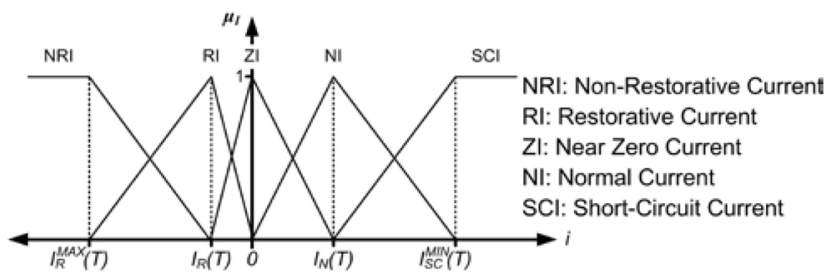

(a)

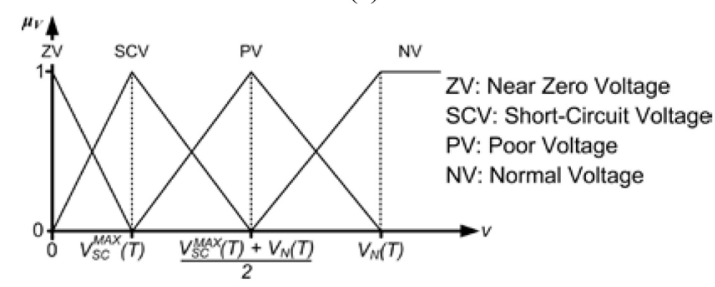

(b)

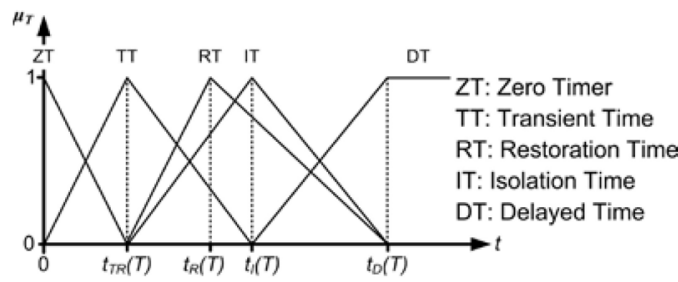

(c)

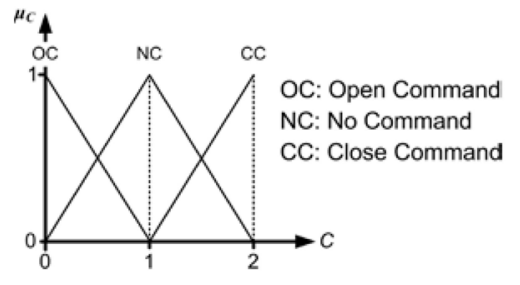

(d)

Fig. 3. Membership functions: (a) current flow; (b) voltage profile; (c) time signal; and (d) command signal.

the time of delayed actuation, when the main switching agent fails; thus, the backup switching agent can act within a fraction of a second during self-healing procedures. Finally, command membership functions incorporate command signals to open and close the switchgear through a remote controller that can be a self-healing coordinator agent or another local agent.

Most membership functions have a triangular shape and can be generically represented by the mathematical model given by (1).

$$
\mu_{G}(x, a, b, c)= \begin{cases}0, & x<a \\ \frac{x-a}{b-a}, & a \leq x<b \\ \frac{x-c}{b-c}, & b \leq x<c \\ 0, & x \geq c\end{cases}
$$

In (1), $\mu_{G}$ is a generic membership function of the $x$ independent variable where $a, b$ and $c$ are scalar parameters. This model is employed to represent mathematically all membership functions of the switching local agent, e.g., the membership function of the isolation time analogously corresponds to $\mu_{I T}\left(t, t_{T R}(T), t_{I}(T), t_{D}(T)\right)$.

The mapping of the monitoring values in linguistic variables comprises the fuzzification procedure. The next step of

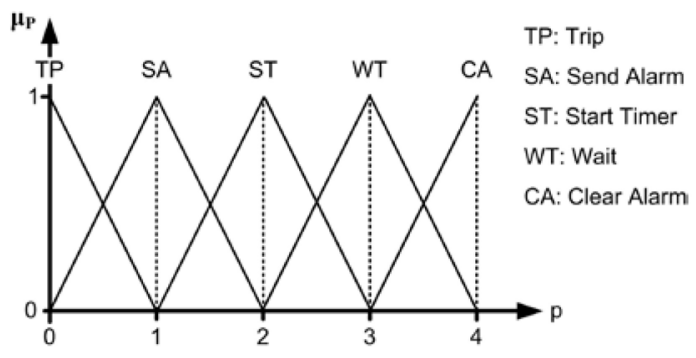

Fig. 4. Numeric response mapped in linguistic variables.

TABLE I

SET OF PRIORITy RULES

\begin{tabular}{|c|c|c|}
\hline \multirow{3}{*}{ 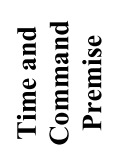 } & $O C$ & TP \\
\hline & $C C$ & CA \\
\hline & $T T$ & WT \\
\hline
\end{tabular}

the fuzzy controller is the inference procedure that generates a numeric response using mapped values and a set of rules. The numeric response is also obtained from linguistic variables as shown by Fig. 4 .

Response linguistic variables are decisions made by the switching agent. The linguistic variable, ST, is a response function used to start the timer that is stopped at zero until the moment of an abnormal measurement. The waiting function, $W T$, is essential for the differentiation between transient and permanent faults because the switching agent only performs time counting during the transitory period. If there is a possible permanent fault in the distribution network, the switching agent sends an alarm, $S A$, to the self-healing coordinator agent. Moreover, the switching agent can trigger the switch tripping, $T P$, when the fault happens in an adjacent section. After that, the timer is zeroed and stopped. In case another adjacent switch is tripped, the local agent clears the alarm, $C A$, closing the switch contacts whenever necessary. Those actions are the resulting propositions of the rule sets in Table I, II and III that establish the priority, isolation and restoration rules, respectively.

The isolation premise highlighted in Table II establishes that the agent decides the tripping, $T P$, when there is no command signal, $N C$, the timer is on isolation time, $I T$, the current flow has a short-circuit magnitude, $S C I$, and the voltage profile has a short-circuit magnitude, $S C V$. This premise is mathematically modeled by (2), while its resulting proposition is given by (3).

$$
\begin{aligned}
r_{k}(i, v, t, c) & =\min \left\{\mu_{I T}, \mu_{N C}, \mu_{S C I}, \mu_{S C V}\right\} \\
P_{k}\left(\mu_{P}\right) & =\left\{p \mid \mu_{T P}=1\right\}
\end{aligned}
$$

The value of the $\mathrm{k}^{\text {th }}$ rule, $r_{k}(i, v, t, c)$, is calculated using instantaneous measures of current flow, $i$, voltage profile, $v$, time, $t$, and command, $c$, while the calculation of the $\mathrm{k}^{\text {th }}$ resulting proposition, $P_{k}\left(\mu_{P}\right)$, uses the resulting membership functions, $\mu_{P}$. Both values are used by the fuzzy controller in 
TABLE II

SET OF ISOLATION RULES

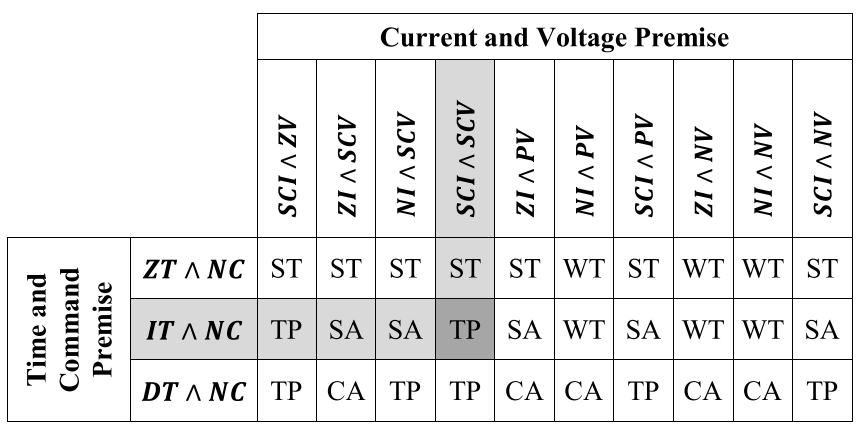

TABLE III

SET OF RESTORATION RULES

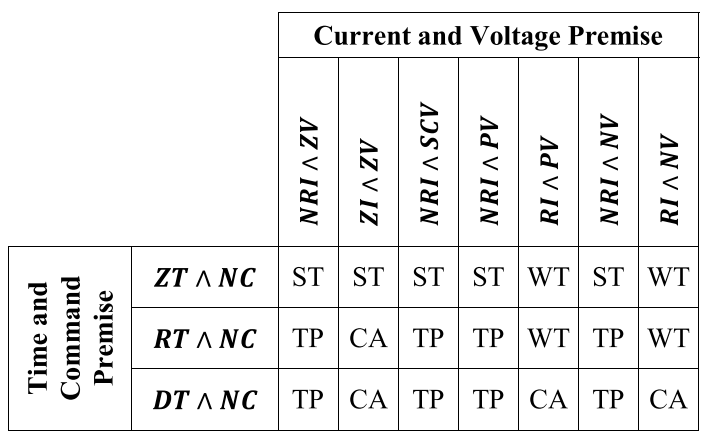

the defuzzification procedure given by (4).

$$
p_{C}\left(i, v, t, c, \mu_{P}\right)=\frac{\sum_{k=1}^{n_{r}} r_{k}(i, v, t, c) P_{k}\left(\mu_{P}\right)}{\sum_{k=1}^{n_{r}} r_{k}(i, v, t, c)}
$$

The defuzzified value, $p_{C}\left(i, v, t, c, \mu_{P}\right)$, also called the fuzzy centroid, is the numeric response of the fuzzy controller and must determine the decisions made by the switching local agent. Furthermore, the switching agent continuously adapts itself through the adjustment of its membership functions using updated control parameters from the self-healing coordinator agent at the intelligence layer. Those control parameters are obtained using the distribution network state.

\section{Analysis Agent for State Estimation}

Agents in the analysis layer give support to the coordinator agents in the intelligence layer through the processing of historical and real-time data provided by agents in the lower layers. For example, the analysis agent can employ the Hamiltonian path theory and Kirchhoff's laws to produce a calculation scheme that allows the real-time estimation of the unknown states using the metering data from local agents [17], [18]. The addition of the calculated network states with the information from the energy market produces a global view of the distribution network that is utilized in load forecasting to obtain the normal, short-circuit and restorative operation conditions. These foreseen scenarios are essential to algorithms of the self-healing coordinator agent such as the isolation and restoration optimization algorithm.

\section{Self-Healing Coordinator Agent}

The self-healing coordinator agent ensures suitable control decisions to maximize system efficiency and minimize the negative impacts of faults. The decision maker acquires data related to the system state and determines a feasible set of control actions proactively in response to three fundamental questions: what is happening, what may happen, and what should be done. The response to these questions encourages the modification of the coordinator agent architecture that can be built using a perception subsystem, which situates the coordinator agent in the environment, with an intelligence subsystem, which manages different aspects of information processing to solve individual and social problems, and with an action subsystem, which sends the control messages to other system agents [11].

The specialization of the self-healing coordinator agent is reached by the functions performed on the intelligence subsystem that respond to the fundamental questions of decision support:

1) Power Flow Calculation: it is related to the first question (What is happening?), and does it determine the normal operating conditions using the information from agents in lower layers;

2) Short-Circuit Simulation: it tries to respond to the second question (What may happen?) through the simulation of probable emergency states for several types of contingencies;

3) Isolation and Restoration Optimization: it focuses on the third question (What should be done?) and aims at the minimization of the operation time of the switching local agents that are subjected to protection and restoration constraints.

Isolation and restoration optimization is the most challenging function because a conventional methodology is not available in the literature, such as a power flow calculation [19] and a short-circuit simulation [20]. Problems with the optimal coordination of protection devices and distribution network restoration are traditionally addressed separately, e.g., procedures for protection coordination and problem formulation are described by [21] and [22], respectively, whereas a method for distribution network restoration is found in [23]. This paradigm should change once the coordinator agent provides control parameters to the switching local agents that act simultaneously to protect and restore the distribution network. Therefore, the new paradigm requires a mathematical formulation to address these self-healing procedures.

$$
\min t_{S S D}(T)=\sum_{j=1}^{n_{S}}\left[t_{I, j}(T)+t_{R, j}(T)\right]
$$

Subject to:

$$
\begin{array}{ll}
t_{D, j}(T)-t_{I, j}(T) \geq C T I, & j=1 \text { to } n_{S}(6) \\
t_{D, j}(T)=\frac{\alpha_{j}(T)}{\left(\frac{\max _{1 \leq k \leq n_{D S}}\left\{I_{S C, k}^{M I N}(T)\right\}}{I_{N, j}(T)}\right)-1}+t_{T R}, & j=1 \text { to } n_{S}
\end{array}
$$




$$
\begin{array}{ll}
t_{I, j}(T)>t_{T R}, & j=1 \text { to } n_{S} \\
t_{R, j}(T)<t_{D, j}(T), & j=1 \text { to } n_{S} \\
t_{R, j}(T)=\frac{I_{R, j}(T)}{I_{R, l}^{M A X}(T)} \beta_{l}+t_{T R}, & j=1 \text { to } n_{S}, l=1 \text { to } n_{F}
\end{array}
$$

In (5), the main objective of the self-healing optimization problem is stated as the minimization of the operation time, $t_{S S D}(T)$, of each switching local agent in the distribution network. The objective function aims at the minimization of the summation of isolation time $t_{I, j}(T)$ and restoration time, $t_{R, j}(T)$ because each $j^{\text {th }}$ switching agent can act to isolate the section with the fault or restore other sections to different adjacent feeders. Similar to a traditional formulation, the optimal coordination of $n_{S}$ switching agents is reached by using a set of constraints, given by (6) to (10).

Equations (6) to (8) ensure the suitable operation of switching local agents during direct power flow. In (6), the difference between instantaneous, $t_{I, j}(T)$, and delayed, $t_{D, j}(T)$, isolation times is limited by the coordination time interval, CTI; thus, the backup switching agents operate whenever the main switching agent fails. In the calculation of $t_{D, j}(T)$, it is assumed that the short-circuit current, $I_{S C, k}^{M I N}(T)$, of the main switch flows in the backup switch as given by (7) and if there are $n_{D S}$ backup switches, the short-circuit current is the maximum value among them. Thus, in (8), the value of $t_{I, j}(T)$ is greater than the transient time, $t_{T R}$, to avoid the switching operation in transitory events.

The coupling between isolation and restoration operations is given by (9) that establishes $t_{D, j}(T)$ as the upper limit of the instantaneous restoration time, $t_{R, j}(T)$. Equation (10) expresses the calculation of $t_{R, j}(T)$ where the relation between the restoration current flowing by the $j^{\text {th }}$ switch, $I_{R, j}(T)$, and the maximal restoration current provided by the adjacent feeder, $I_{R, l}^{M A X}(T)$, is predominant. Both restoration currents result from the solution of a combinatorial optimization problem where the distribution network limits the operational range of the voltage profile and the maximum capacity of power transference must not be violated [23].

Fig. 5 demonstrates the self-healing coordination procedure through a single-line diagram of a distribution network section that reveals the probable direct and reverse power flows. In direct flow, the substation provides power to the distribution network and $I_{N, A}(T)$ flows through switch A during the normal operating condition. If a short-circuit occurs, switches A and B actuates to isolate the fault according with the intensity of the short-circuit current. The chart in (b) shows the characteristic curve for isolation coordination thus the switch A must actuate in $t_{D, A}(T)$ whenever the fault happens in the section limited by the switch B because the intensity of $I_{S C, B}^{M I N}(T)$ is less than $I_{S C, A}^{M I N}(T)$. This isolation feature is well known in specialized literature where the coordination between main and backup switches depends on the $C T I$ value. Immediately after the interruption of direct flow, the reverse power flow arises in the isolated sections due to the restoration process that is begun with the closing of the tie switch connected to the $\mathrm{j}^{\mathrm{t}}$ adjacent feeder. The chart in (c) presents the characteristic used to

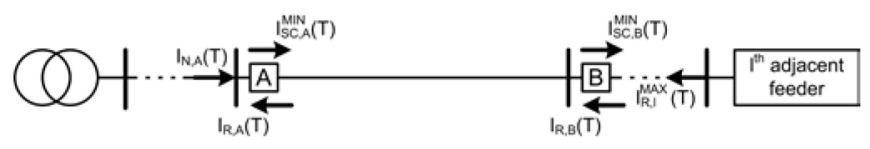

(a)

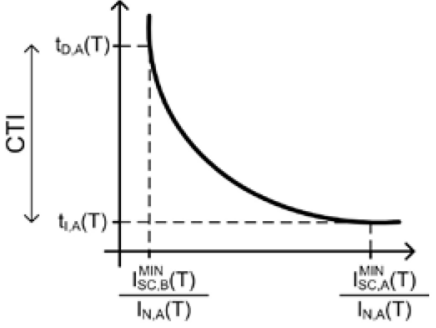

(b)

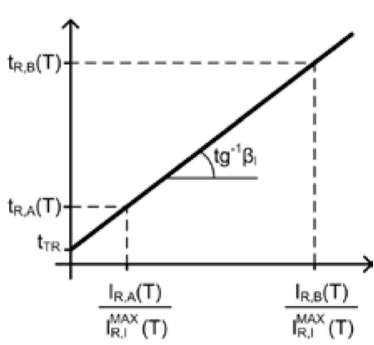

(c)
Fig. 5. Coordination procedure of two switching local agents: (a) single-line diagram; (b) isolation and; (c) restoration coordination.

coordinate the switching local agents during the procedure of automatic restoration that is not conventional and determines the opening of the distant switches before the switches close to the adjacent feeder according to their restoration current magnitudes, i.e., when the reverse current is bigger than $I_{R, l}^{M A X}(T)$, the switch A must actuates before the switch B because the intensity of $I_{R, A}(T)$ is less than $I_{R, B}(T)$.

\section{Study CASE: Distribution Network Behavior}

The proposed methodology was investigated over a real distribution network that has its data available in [24], and its topology is according to the single-line diagram in Fig. 6. The testing distribution network has nine switches from SW1 to SW9 and five tie switches from TS1 to TS5 in connection with neighbor feeders. Furthermore, each switch is controlled by a switching local agent. The performed tests aim to study the coordination algorithm of the self-healing coordinator agent and to check the fuzzy controller through the behavior analyses of membership functions and numeric responses of switching local agents.

An investigation of the isolation and restoration optimization algorithm focuses on calculations of scalar parameters sent to the switching agents. Table IV presents the adjustment parameters produced by the coordination algorithm at midday for all switching agents in the distribution network. These results were obtained using $C T I=0.3 \mathrm{~s}, t_{T R}=0.1 \mathrm{~s}$, $0 \leq \alpha_{j} \leq 1$ and $0.1 \geq \beta_{l} \geq 10$; moreover, the short-circuit parameters were calculated to a fault of high impedance $(50 \Omega)$ in the most distant termination of each section.

The highlighted adjustment parameters reveal the expected results of the coordination algorithm for two adjacent switching agents, SW4 and SW5, as illustrated by Fig. 5, where the farthest switch must have a delayed action in $30 \mathrm{~ms}$ during the restoration procedure. In the performed tests, the coordination algorithm obtained adjustment parameters hourby-hour to update the scalar parameters of the membership functions.

Fig. 7 shows the behavior of the membership functions of current flow and the voltage profile hour-by-hour during a day for SW5, where the cold color tones indicate membership 


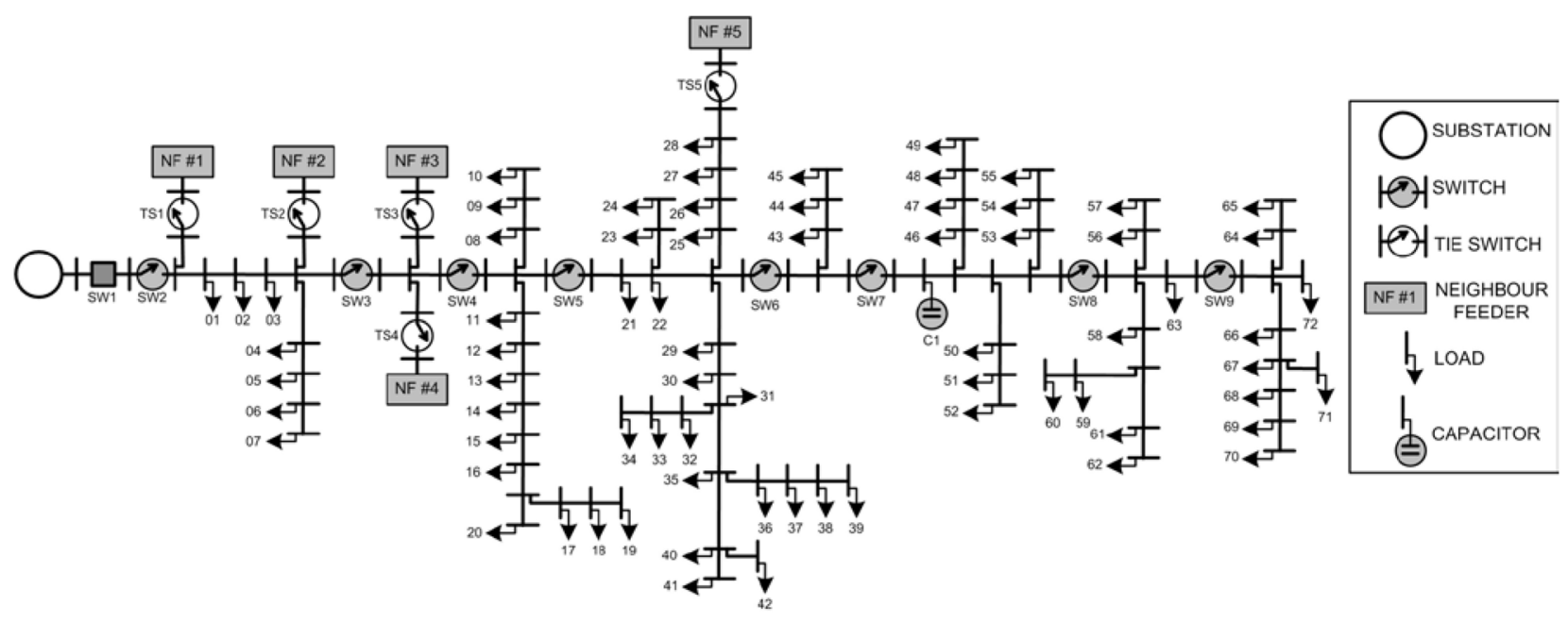

Fig. 6. Simplified topological diagram of the real distribution network used in the methodology tests.

TABLE IV

Adjustment Parameters Produced by the Self-Healing Coordinator Agent at Midday (T=12:00)

\begin{tabular}{|c|c|c|c|c|c|c|c|c|c|c|}
\hline ID & CLOSED & $V_{N, j}(T)$ & $V_{S C, j}^{M A X}(T)$ & $I_{R, j}^{M A X}(T)$ & $I_{R, j}(T)$ & $I_{N, j}(T)$ & $I_{S C, j}^{M I N}(T)$ & $t_{I, j}(T)$ & $t_{D, j}(T)$ & $t_{R, j}(T)$ \\
\hline SW1 & True & $7960.00 \mathrm{~V}$ & $7960.00 \mathrm{~V}$ & $-96.87 \mathrm{~A}$ & $0.00 \mathrm{~A}$ & $170.03 \mathrm{~A}$ & $320.52 \mathrm{~A}$ & $0.2130 \mathrm{~s}$ & $0.5130 \mathrm{~s}$ & $0.1000 \mathrm{~s}$ \\
\hline SW2 & True & $7958.12 \mathrm{~V}$ & $7957.32 \mathrm{~V}$ & $-96.87 \mathrm{~A}$ & $0.00 \mathrm{~A}$ & $170.03 \mathrm{~A}$ & $316.60 \mathrm{~A}$ & $0.2160 \mathrm{~s}$ & $0.5160 \mathrm{~s}$ & $0.1000 \mathrm{~s}$ \\
\hline SW3 & True & $7803.47 \mathrm{~V}$ & $7736.71 \mathrm{~V}$ & $-96.87 \mathrm{~A}$ & $-14.90 \mathrm{~A}$ & $155.17 \mathrm{~A}$ & $303.90 \mathrm{~A}$ & $0.2043 \mathrm{~s}$ & $0.5043 \mathrm{~s}$ & $0.1154 \mathrm{~s}$ \\
\hline SW4 & True & $7764.65 \mathrm{~V}$ & $7675.94 \mathrm{~V}$ & $-96.87 \mathrm{~A}$ & $-14.90 \mathrm{~A}$ & $155.17 \mathrm{~A}$ & $301.44 \mathrm{~A}$ & $0.2061 \mathrm{~s}$ & $0.5061 \mathrm{~s}$ & $0.1154 \mathrm{~s}$ \\
\hline SW5 & True & $7757.55 \mathrm{~V}$ & $7672.03 \mathrm{~V}$ & $-96.87 \mathrm{~A}$ & $-41.06 \mathrm{~A}$ & $129.17 \mathrm{~A}$ & $274.16 \mathrm{~A}$ & $0.1891 \mathrm{~s}$ & $0.4891 \mathrm{~s}$ & $0.1424 \mathrm{~s}$ \\
\hline SW6 & True & $7694.87 \mathrm{~V}$ & $7565.93 \mathrm{~V}$ & $-100.00 \mathrm{~A}$ & $0.00 \mathrm{~A}$ & $73.46 \mathrm{~A}$ & $223.11 \mathrm{~A}$ & $0.1491 \mathrm{~s}$ & $0.4491 \mathrm{~s}$ & $0.1000 \mathrm{~s}$ \\
\hline SW7 & True & $7691.98 \mathrm{~V}$ & $7555.37 \mathrm{~V}$ & $-100.00 \mathrm{~A}$ & $0.00 \mathrm{~A}$ & $67.43 \mathrm{~A}$ & $216.68 \mathrm{~A}$ & $0.1452 \mathrm{~s}$ & $0.4452 \mathrm{~s}$ & $0.1000 \mathrm{~s}$ \\
\hline SW8 & True & $7661.08 \mathrm{~V}$ & $7495.68 \mathrm{~V}$ & $-100.00 \mathrm{~A}$ & $0.00 \mathrm{~A}$ & $44.65 \mathrm{~A}$ & $191.11 \mathrm{~A}$ & $0.1305 \mathrm{~s}$ & $0.4305 \mathrm{~s}$ & $0.1000 \mathrm{~s}$ \\
\hline SW9 & True & $7653.27 \mathrm{~V}$ & $7459.44 \mathrm{~V}$ & $-100.00 \mathrm{~A}$ & $0.00 \mathrm{~A}$ & $21.48 \mathrm{~A}$ & $169.44 \mathrm{~A}$ & $0.1145 \mathrm{~s}$ & $0.4145 \mathrm{~s}$ & $0.1000 \mathrm{~s}$ \\
\hline TS1 & False & $7955.46 \mathrm{~V}$ & $7947.04 \mathrm{~V}$ & $-14.90 \mathrm{~A}$ & $-14.90 \mathrm{~A}$ & $0.00 \mathrm{~A}$ & $0.00 \mathrm{~A}$ & $0.1000 \mathrm{~s}$ & $0.4000 \mathrm{~s}$ & $0.2000 \mathrm{~s}$ \\
\hline TS2 & False & $7898.05 \mathrm{~V}$ & $7786.67 \mathrm{~V}$ & $-14.90 \mathrm{~A}$ & $-14.90 \mathrm{~A}$ & $0.00 \mathrm{~A}$ & $0.00 \mathrm{~A}$ & $0.1000 \mathrm{~s}$ & $0.4000 \mathrm{~s}$ & $0.2000 \mathrm{~s}$ \\
\hline TS3 & False & $7875.50 \mathrm{~V}$ & $7720.42 \mathrm{~V}$ & $-14.90 \mathrm{~A}$ & $-14.90 \mathrm{~A}$ & $0.00 \mathrm{~A}$ & $0.00 \mathrm{~A}$ & $0.1000 \mathrm{~s}$ & $0.4000 \mathrm{~s}$ & $0.2000 \mathrm{~s}$ \\
\hline TS4 & False & $7875.50 \mathrm{~V}$ & $7720.42 \mathrm{~V}$ & $-14.90 \mathrm{~A}$ & $-14.90 \mathrm{~A}$ & $0.00 \mathrm{~A}$ & $0.00 \mathrm{~A}$ & $0.1000 \mathrm{~s}$ & $0.4000 \mathrm{~s}$ & $0.2000 \mathrm{~s}$ \\
\hline TS5 & False & $7831.78 \mathrm{~V}$ & $7608.40 \mathrm{~V}$ & $-96.87 \mathrm{~A}$ & $-96.87 \mathrm{~A}$ & $0.00 \mathrm{~A}$ & $0.00 \mathrm{~A}$ & $0.1000 \mathrm{~s}$ & $0.4000 \mathrm{~s}$ & $0.2000 \mathrm{~s}$ \\
\hline
\end{tabular}

values near to zero and hot tones indicate values near to one. In (a), the time fluctuations of the membership functions in Fig. 3(a) are plotted using a surface chart revealing that the non-restorative current, NRI, varies from $65 \mathrm{~A}$ at the early day to around of 100A after the midday, analogously, the restorative current, $R I$, presents a fluctuation range of 22A over day. The normal current, NI, and short-circuit current, SCI, also fluctuate continuously over day, particularly at period between 16 and 18 hours, when the variations are more evident. In (b), the surface chart of the time adjustment of the membership functions in Fig. 3(b) shows more intense fluctuations. The fluctuation range of the short-circuit voltage profile, $S C V$, poor voltage profile, $P V$, and normal voltage profile, $N V$, are overlapped but the features of these membership functions remain the same for each hour. Thus, the obtained awareness regarding operating conditions of the distribution network can aid the switching local agents in making more accurate and flexible decisions when a contingency is occurring in the distribution network.
After checking the membership functions, the performance of the proposed self-healing strategy was evaluated from the distribution network behavior to the occurrence of a fault in the section limited by switches SW3 and SW4. Numerical results plotted into time charts are shown in Fig. 8 and reveal the actions taken by the SW3, SW4 and TS5 switches to isolate the faulty section and restore other affected sections. The fault happens at $t=0.1 \mathrm{~s}$ and is marked by an abrupt increase in current flow and defines the instant when all of the observed switching agents start their timers. SW3 is the first switching agent that makes the decision of opening its contacts at $t=0.28 \mathrm{~s}$ to protect the main source. Immediately after, the TS5 tie-switch closes its contacts due to absence of power source that, also, retains the conditions to SW4 and SW5 clear the alarm. In this time, an intense reverse current flow circulates through SW4 and SW5 when their switching agents start the timers again. After $0.1 \mathrm{~s}$, the SW4 switching agent makes the decision to tripping that reduces the reverse flow to a normal level in the distribution network. 


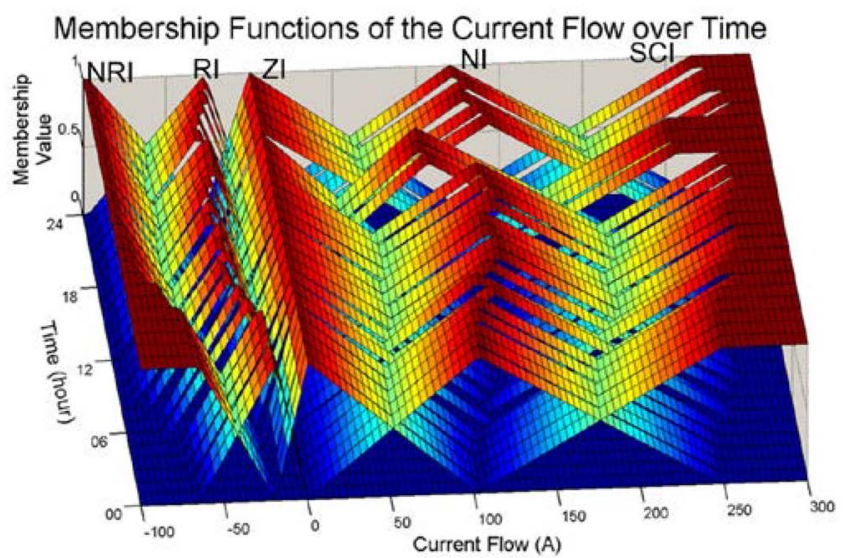

(a)

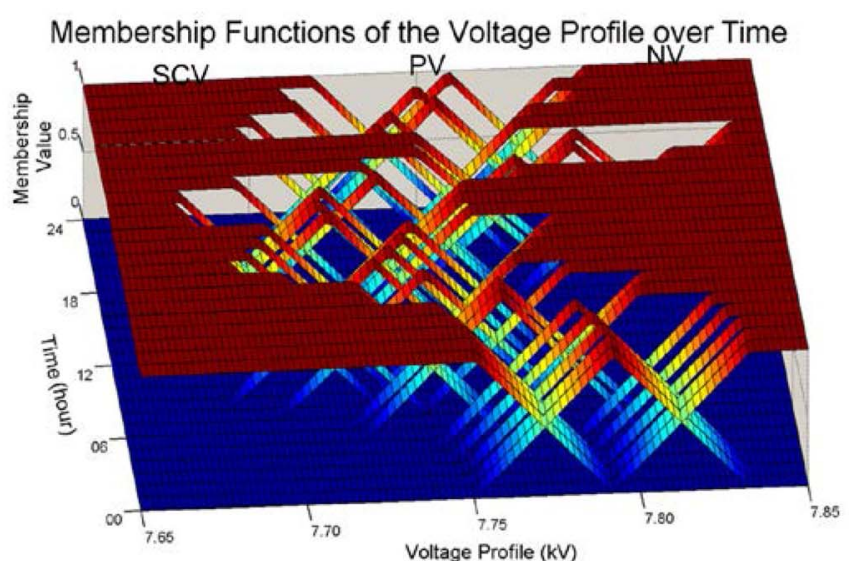

(b)

Fig. 7. Surface charts with the time variation of the membership functions for SW5: (a) current flow and; (b) voltage profile.
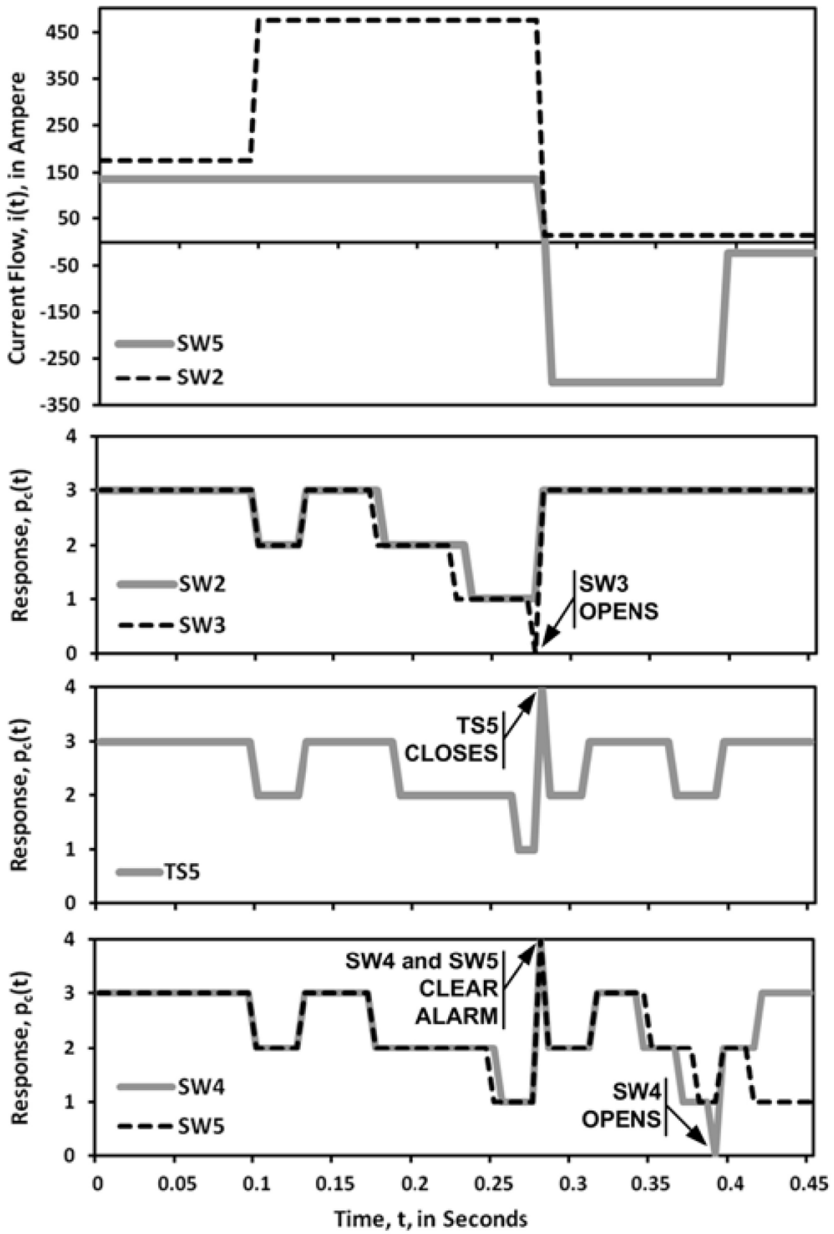

Fig. 8. Numerical responses of switching local agents over time.

The success of the proposed self-healing strategy comes from the proactivity of the MAS where the self-healing coordinator agent proactively modifies the perception of all switching local agents. In this way, when a probable contingency occurs in the distribution network, the distributed switching agents are ready and actuate cooperatively among them to clear the contingency without any centralized assistance, as shown by the results.

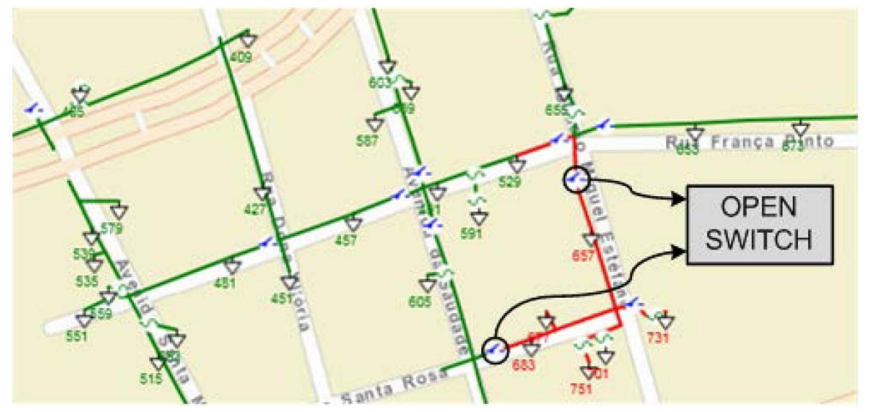

Fig. 9. Response of the dynamic coloring tool to the line section isolation.

\section{A. Location of the Faulted Line-Section}

The fault indicator can generally reduce operating costs and service interruptions by identifying de faulted line sections [25]. In this way, proposed self-healing strategy should be integrated with the dynamic coloring tool that permits to the system operator monitor the energy quality delivered to the end consumers using the monitoring of the voltage profile [26]. The dynamic coloring tool uses several resources from the advanced distribution management system such as geo-referenced topological diagrams in multiple layers and the results of the real-time state estimation that employs the smart meter data [17], [18]. If the voltage ranges are standardized and associated with a specific color, each drawn branch should have a color in the topological diagram in accord with the voltage profile provided by the state estimation.

Fig. 9 shows a piece of the topological diagram drawn using the dynamic coloring tool where the line section, which is limited by two opened switches, is plotted in red color because the smart meters in this isolated line section measures a voltage profile equals to zero. Thus, the localization of the affected line section can be readily known during fault events because the dynamic coloring tool updates the topological diagram in each time interval improving the decision-making process that also employs alarm signals sent by the switching agents. 


\section{B. Important Issues About the Communication Channel}

The IEC 61850 standard defines ten communication interfaces, of which one determines the control-data exchange between the substation and remote control center, and another one indicates the direct data exchange among the bay units for fast functions [14]. The first interface enables the hierarchical communication between the coordinator agent and switching agents via control service model, while the second interface is employed in the peer-to-peer real-time communication among switching agents through the generic object oriented substation events (GOOSE) messages. The utilization of different communication interfaces contributes for avoiding the conflict of the agent messages; moreover parallel messages are ranked according the priority of each switching agent.

In a scenario of communication loss among agents of upper and lower layers, a secondary protection scheme is enabled because the coordinator agent cannot send the adjustment parameters to the switching agents that, now, use a set of default adjustment parameters stored in their programming memory. Until the reestablishment of the communication channel, the switching agents only work for the maximum operational limit of the distribution network that reduces the accuracy and flexibility of switching agents.

\section{CONCLUSION}

Different from many methodologies found in literature that centralize restoration algorithms in the operation center where the switching sequence is determined and is sent to switching devices via a communication network during the restoration procedure, the proposed automation strategy employs the techniques of DAI. This technique enables the construction of the MAS architecture to achieve real self-healing behavior with a fast isolation and restoration of the distribution network. In the proposed MAS structure, the switching local agent aggregates the local intelligence and the distributed intelligence characteristics; thus, the switching local agent can isolate the nearest fault and still be aware of the whole distribution network by updating the control parameters that modify its internal functions in each time interval. The continuous updating of the control parameters increases the selectivity of the switching agents for the self-healing structure. Thus, short circuits of high impedance, which are hardly sensed by conventional protection devices, are easily detected by the switching local agents.

\section{REFERENCES}

[1] S. Borlase, "Smart grid technologies," in Smart Grids: Infrastructure, Technology and Solutions, vol. 1, 1st ed. Boca Raton, FL, USA: CRC Press, 2013, pp. 67-494.

[2] A. Bernardo, N. Silva, A. Carrapatoso, and G. Ockwell, "Preventive assessment for combined control centre and substation-centric selfhealing strategies," in Proc. 21st Int. Conf. Elect. Distrib. (CIRED), Frankfurt, Germany, 2011, pp. 1-4.

[3] C. McCarhy, M. Edmonds, A. Jayantilal, and E. Boardman, "Smart distribution through layered intelligence for next generation self-healing distribution networks," in Proc. 22nd Int. Conf. Elect. Distrib. (CIRED), Stockholm, Sweden, 2013, pp. 1-4.

[4] L. Maurer, A. Stevens, and W. Reder, "Tales from the Frontline: Keys to successful self-healing distribution projects," IEEE Power Energy Mag., vol. 10, no. 2, pp. 100-106, Mar./Apr. 2012.

[5] G. Weiss, Multiagent Systems: A Modern Approach to Distributed Artificial Intelligence. Cambridge, MA, USA: MIT Press, 1999.
[6] M. Wooldridge, "Intelligent agents," in Multiagents Systems: A Modern Approach to Distributed Artificial Intelligence. Cambridge, MA, USA: MIT Press, 1999, pp. 27-78.

[7] M. N. Huhns and L. M. Stephens, "Multiagent systems and societies of agents," in Multiagents Systems: A Modern Approach to Distributed Artificial Intelligence. Cambridge, MA, USA: MIT Press, 1999, pp. 79-120.

[8] A. Zidan and E. F. El-Saadany, "A cooperative multiagent framework for self-healing mechanisms in distribution systems," IEEE Trans. Smart Grid, vol. 3, no. 3, pp. 1525-1539, Sep. 2012.

[9] H. Liu, X. Chen, K. Yu, and Y. Hou, "The control and analysis of self-healing urban power grid," IEEE Trans. Smart Grid, vol. 3, no. 3, pp. 1119-1129, Sep. 2012.

[10] F. Ding and K. A. Loparo, "Hierarchical decentralized network reconfiguration for smart distribution systems-Part I: Problem formulation and algorithm development," IEEE Trans. Power Syst., vol. 30, no. 2, pp. 734-743, Mar. 2015.

[11] J. Cuena and S. Ossowski, "Distributed models for decision support," in Multiagents Systems: A Modern Approach to Distributed Artificial Intelligence. Cambridge, MA, USA: MIT Press, 1999, pp. 459-504.

[12] J. B. Leite and J. R. S. Mantovani, "Development of a smart grid simulation environment, part I: Project of the electrical devices simulator,' J. Control Autom. Elect. Syst., vol. 26, no. 1, pp. 80-95, Feb. 2015.

[13] Communication Networks and Power Systems in Substations, IEC Standard 61850-1, Oct. 2003.

[14] J. B. Leite and J. R. S. Mantovani, "Study of the IEC 61850 protocol on multiagent systems for power system applications," in Proc. IEEE PES Gen. Meeting, Denver, CO, USA, 2015, pp. 1-5.

[15] D. L. Poole and A. K. Mackworth, "Agent architectures and hierarchical control," in Artificial Intelligence: Foundations of Computational Agents, 1st ed. Cambridge, U.K.: Cambridge Univ. Press, 2010, pp. 43-68.

[16] F. M. Mcneill and E. Thro, "Fuzzy systems on the job," in Fuzzy Logic: A Practical Approach, 1st ed. Boston, MA, USA: Academic Press, 1994, pp. 57-82.

[17] J. B. Leite and J. R. S. Mantovani, "State estimation of distribution networks through the real-time measurements of the smart meters," in Proc. IEEE PES PowerTech, Grenoble, France, 2013, pp. 1-6.

[18] J. B. Leite and J. R. S. Mantovani, "Distribution system state estimation using the hamiltonian cycle theory," IEEE Trans. Smart Grid, vol. 7, no. 1, pp. 366-375, Jan. 2016

[19] C. S. Cheng and D. Shirmohammad, "A three-phase power flow method for real-time distribution system analysis," IEEE Trans. Power Syst., vol. 10, no. 2, pp. 671-679, May 1995.

[20] X. Zhang, F. Soudi, D. Shirmohammad, and C. S. Cheng, "A distribution short circuit analysis approach using hybrid compensation method," IEEE Trans. Power Syst., vol. 10, no. 4, pp. 2053-2059, Nov. 1995.

[21] P. M. Anderson, "Fault protection of radial lines," in Power System Protection, vol. 1, 1st ed. Hoboken, NJ, USA: Wiley, 1999, pp. 201-240.

[22] B. Chattopadhyay, M. S. Sachdev, and T. S. Sidhu, "An on-line relay coordination algorithm for adaptive protection using linear programming technique," IEEE Trans. Power Del., vol. 11, no. 1, pp. 165-173, Jan. 1996.

[23] J. Li, X.-Y. Ma, C.-C. Liu, and K. P. Schneider, "Distribution system restoration with microgrids using spanning tree search," IEEE Trans. Power Syst., vol. 29, no. 6, pp. 3021-3029, Nov. 2014.

[24] Distribution Testing System of 363 Lines. [Online]. Available: http://www.feis.unesp.br/Home/departamentos/engenhariaeletrica/ lapsee807/home/distribution_network_363_lines.rar, accessed Jul. 3, 2015

[25] J.-H. Teng, W.-H. Huang, and S.-W. Luan, "Automatic and fast faulted line-section location method for distribution systems based on fault indicators," IEEE Trans. Power Syst., vol. 29, no. 4, pp. 1653-1662, Jul. 2014.

[26] J. B. Leite and J. R. S. Mantovani, "Development of a smart grid simulation environment, part II: Implementation of the advanced distribution management system," J. Control Autom. Elect. Syst., vol. 26, no. 1, pp. 96-104, Feb. 2015.

Jônatas Boás Leite (S'10-M'15) received the B.Sc. and Ph.D. degrees in electrical engineering from UNESP, Ilha Solteira, Brazil, in 2010 and 2015, respectively. He is currently a Postdoctoral Researcher with the Electrical Engineering Post-Graduate Program, UNESP. His research areas are planning and control of electric power systems.

José Roberto Sanches Mantovani (M'06) received the B.Sc. degree from UNESP, Ilha Solteira, Brazil, in 1981, and the M.S. and Ph.D. degrees in electrical engineering from UNICAMP, Campinas, Brazil, in 1987 and 1995, respectively. He is currently a Professor with the Electrical Engineering Department, UNESP. His research areas are planning and control of electric power systems 